\title{
Use of the non-radioactive SUnSET method to detect decreased protein synthesis in proteasome inhibited Arabidopsis roots
}

\author{
Doug Van Hoewyk $k^{1,2^{*}}$
}

\begin{abstract}
Background: In eukaryotic cells, the proteasome maintains homeostasis by selectively degrading regulatory and misfolded proteins, and in doing so contributes to the amino acid pool. Inhibition of the proteasome in yeast and human cells decreases de novo protein synthesis. However, it is not know if proteasome inhibition in plants similarly suppresses protein synthesis. To address this gap in plant biology, protein synthesis in Arabidopsis roots was estimated using SUface SEnsing of Translation (SUnSET) techniques. This non-radioactive method has been validated in animal cells, but has not yet been applied to plants. The goal of this study was to investigate the suitability of SUnSET methodology to measure protein synthesis in plants, and to determine if proteasome inhibition decreases levels of newly synthesized proteins.

Results: The SUnSET technique revealed that Arabidopsis plants treated with cycloheximide —an inhibitor of protein synthesis - severely decreased levels of newly synthesized proteins in root and shoot tissue, as detected on a Western Blot. Therefore, the non-radioactive method is suitable to detect changes in protein synthesis, and was subsequently used to monitor protein synthesis in proteasome-inhibited roots. The proteasome inhibitor MG132 decreased levels of newly synthesized proteins by $70-80 \%$ after 4 and $16 \mathrm{~h}$. Removal of MG132 from liquid media resulted in roots with increased levels of newly synthesized proteins compared to untreated plants, suggesting that recovery from proteasome inhibition results in elevated levels of protein synthesis. Additionally, SUnSET was used to detect a decrease in protein synthesis in the roots of plants subjected to salt stress or sulfur starvation.

Conclusions: Proteasome inhibition has been shown to decrease protein synthesis in yeast and human cells, and this study now shows that MG132's inhibitory effects also applies to plants. These data represent the first time that SUnSET has been used to measure protein synthesis in plants. The study demonstrates that SUnSET is a suitable and robust technique to measure protein synthesis in plants. The use of this non-radioactive method to gauge protein synthesis offers a fast, safe, and cost-effective alternative compared to traditional techniques that rely upon radioactive material. The method is likely to have broad applicability to different disciplines in plant biology.
\end{abstract}

\section{Background}

The ubiquitin proteasome pathway (UPP) is found in all eukaryotic cells and functions by selecting ubiquitinated proteins for proteasomal degradation in the cytosol or nucleus [1]. Proteolysis of ubiquitinated proteins by the proteasome serves a variety of functions. The proteasome

\footnotetext{
*Correspondence: dougvh@coastal.edu

${ }^{2}$ Present Address: Ankara University, Biotechnology Institute, Tandoğan

Campus, 06110 Ankara, Turkey

Full list of author information is available at the end of the article
}

degrades many short-lived regulatory proteins, including those governing in cell division [2]. In plants, the UPP can also regulate nutrient status [3] and hormone signaling [4]. The proteasome also clears the cell of oxidized or misfolded proteins that result from stress, thereby preventing toxic protein aggregation. In addition to the removal of regulatory and damaged proteins, it is estimated that about $30 \%$ of newly synthesized proteins might undergo rapid proteasomal degradation [5].

The degradation of proteins mediated by the UPP serves to maintain the amino acid pool, but this has only 
been established in non-plant models. Proteasome inhibition in nutrient-deprived human cells depleted the amino acid pool and decreased protein synthesis [6]. More recently, it was reported that amino acid depletion resulting from UPP impairment results in lethality in human cells, yeast, and Drosophila [7]. Predictably, amino acid shortage induced by the proteasome inhibitor MG132 rapidly decreases de novo protein synthesis. In mouse cells treated with MG132, protein synthesis decreased by $90 \%$ after $6 \mathrm{~h}$ [8]. A nearly similar result, i.e. $80 \%$ decrease in protein synthesis, was observed in yeast with impaired proteasome activity [7]. MG132 has also been reported to decrease protein synthesis in neurons [9] and myoblasts [10].

The consequences of UPP inhibition are well-characterized in yeast and mammalian cells, and in humans is associated with several neurodegenerative diseases [11]. In contrast, our understanding of the consequences of proteasome inhibition in plants lags behind that of yeast and humans. This is unfortunate, given the increasing evidence suggesting that plant proteasomes area susceptible to a variety of stressors. For example, some plant pathogens can release effectors that directly impair the proteasome's catalytic core, which serves to promote pathogenic infection in plants [12]. Evidence is also mounting that abiotic stress impairs the UPP. Decreased proteasome activity in plants can be caused by heavy metals [13-15] and salt stress [16]. Selenite stress also impairs the UPP, which was associated with an accumulation of superoxide in Chlamydomonas [17] and Brassica napus [18]. Plants can respond to proteasome inhibition by initiating autophagy, including the degradation of inactive proteasomes [19]. Despite these recent findings, it still is not known if proteasome impairment in plants decreases de novo protein synthesis.

This study employed SUface SEnsing of Translation (SUnSET) techniques to determine if proteasome inhibition in plants decreases the rate of newly synthesized proteins. This non-radioactive technique was recently reviewed [20], and has been widely employed in human cell lines [21] and human tissue, including skeletal muscle [22] and the hippocampus [23]; however, SUnSET has not been demonstrated in plants. Briefly, this technique requires treatment and uptake of the antibiotic puromycin, a structural analogue of tyrosal-tRNA that contains a non-hydrolyzable bond between the tRNA and amino acid. Incorporation of puromycin into nascent polypeptides causes termination. Although high concentrations of puromycin is toxic because it can inactivate translation, at low concentrations it provides an accurate snapshot of protein synthesis without causing lethality. Levels of newly synthesized proteins containing puromycin and its analogs [24] can be measured by immunohistochemistry, fluorescence activated cell sorting (FACS), and confocal microscopy [24]. Alternatively, the newly synthesized polypeptides can be detected as low-molecular weight bands on a Western-blot using an anti-puromycin antibody, and less cost-prohibitive.

SUnSET has been used to estimate both increased and decreased levels of newly synthesized proteins. For example, SUnSET has been used to detect decreased protein synthesis in cells treated with cycloheximide and arsenite [21], known inhibitors of protein synthesis. Additionally, the technique has also been applied to monitor increased protein synthesis in muscle cells treated with insulin [22]. In both studies, rates of protein synthesis were validated with established radioactive techniques, and changes in protein synthesis were nearly identical when comparing the two methodologies. The goal of this study was twofold. Initial experiments were conducted to determine if SUnSET is a suitable method to gauge changes in protein synthesis, as estimated by Western-blotting. Secondly, SUnSET was employed to decifer if proteasome inhibition decreases the pool of newly synthesized proteins in roots.

\section{Methods}

\section{Growth conditions}

Arabidopsis plants (ecotype Columbia) were germinated on soil, and on day 7 transferred to aerated Hoagland's media. Plants were hydroponically grown for an additional 21 days in a growth chamber $(150 \mu$ Einsteins, $16 \mathrm{~h}$ light $/ 8 \mathrm{~h}$ dark cycle, $24^{\circ} \mathrm{C}$ ) before subjected to additional stress treatments. To inhibit the proteasome, plants were transferred to $50 \mathrm{~mL}$ of aerated Hoagland's media containing $0.1 \%$ DMSO with or without $50 \mu \mathrm{M}$ MG132, a concentration that is known to impair the proteasome in roots [18]. Plants were treated with MG132 for 0, 4, and $16 \mathrm{~h}$. To induce salt stress, plants were treated with $100 \mathrm{mM} \mathrm{NaCl}$ for 4 day. Plants subjected to sulfur starvation were also grown for an additional 4 day; sulfur deprivation was induced by replacing sulfate-salts in the Hoagland's media with chloride salts.

\section{SUnSET method and electrophoresis}

After stress treatments, Arabidopsis plants were transferred into a $50 \mathrm{~mL}$ test tube containing $20 \mathrm{~mL}$ of Hoagland's media with or without puromycin. Puromycin is toxic and inhibits protein synthesis at high concentrations. Therefore, to detect protein synthesis in roots, plants were initially treated with 2 and $20 \mu \mathrm{M}$ for $30 \mathrm{~min}$. To estimate protein synthesis in shoots, plants were treated with 20 and $50 \mu \mathrm{M}$ puromycin for 30 and $60 \mathrm{~min}$. Roots and shoots were separated and washed $2 \times$ in distilled water. Plant material was subsequently ground in liquid nitrogen, and a clarified protein extract was obtained for electrophoresis. 
Newly synthesized proteins using the SUnSET method were detected by immunoblotting. To detect the truncated proteins the arise from puromycin's misincorporation into polypeptides, $20 \mu \mathrm{g}$ of protein were loaded onto a $15 \%$ SDS-PAGE gel and run under denaturing conditions. Proteins were transferred on a PVDF membrane. Prior to immunoblotting, membranes were stained with Ponceau Red to ensure an equal loading of proteins. Membranes were subsequently washed, blocked in milk, and incubated for $2 \mathrm{~h}$ with the puromycin antibody (PMY-2A4) purchased from the University of Iowa, USA [25]. The PMY-2A4 anitbody was used at a 1:1000 dilution. Newly synthesized proteins containing puromycin were detected using a secondary antibody conjugated to alkaline phosphatase (1:10,000 dilution for $45 \mathrm{~min})$. Protein synthesis was estimated based on the intensity of immunoreactive bands using image-J.

To confirm that SUnSET was suitable to detect changes in protein synthesis in roots, plants were treated with either 1 or $20 \mu \mathrm{M}$ cycloheximide, a potent inhibitor of protein synthesis. After a $4 \mathrm{~h}$ treatment, protein synthesis in root tissue was estimated using SUnSET as described above.

Accumulation of high molecular weight poly-ubiquitinated proteins was detected on an $8 \%$ SDS-PAGE gel [26]. Cox2, a $30 \mathrm{kDa}$ protein, was analyzed from the same membrane that detected polyubiquitinated proteins. Bip2 (luminal binding protein 2) is $72 \mathrm{kDa}$, and was analyzed from the same membrane that detected newly synthesized proteins. Both Cox2 and Bip2 were analyzed as previously described [18].

\section{Microscopy and amino acid analysis}

Cell viability in the root tips of plants treated with or without MG132 was estimated as similarly performed [18]. Briefly, excised root tips were incubated for $15 \mathrm{~min}$ in $1 \mathrm{~mL}$ of $50 \mathrm{mM}$ Tris buffer, $\mathrm{pH} 7.5$, containing $20 \mu \mathrm{M}$ fluorescein diacetate. Root tips were subsequently washed $5 \times$ in Tris buffer to remove the fluorescent probe. Fluorescence of fluorescein diacetate (optimal Ex495/Em515) is dependent upon cell membrane integrity, and was estimated using a FITC filter set.

Levels of total amino acids were analyzed using a modified ninhydrin method [27]. Glutamic acid was used to make a standard curve, and spectrophotometrically measured at $\mathrm{A}_{570}$. Statistical analyses (ANOVA) were performed using the Kleida-graph software package (Synergy Software).

\section{Results}

SUnSET has successfully been used to estimate changes in protein synthesis in yeast and animal cells, but the technique has not been employed in plants. Therefore, it was necessary to determine the suitability of SUnSET using appropriate controls. Hydroponically grown Arabidopsis plants were treated with puromycin $(0,2$, and $20 \mu \mathrm{M}$ ) for $30 \mathrm{~min}$, and proteins from root tissue were extracted. The intensity of the immunoreactive bands on a Western Blot correlated with puromycin concentration (Fig. 1a). Importantly, untreated control plants did not produce a band. Next, the effects of the cycloheximidea protein synthesis inhibitor-were examined using SUnSET. Plants were treated with cycloheximide $(0,1$, and $20 \mu \mathrm{M})$ for $4 \mathrm{~h}$. After $4 \mathrm{~h}$, roots treated with $1 \mu \mathrm{M}$ cycloheximide exhibited an $80 \%$ decrease in protein synthesis compared to untreated plants; newly synthesized proteins were not detected in Arabidopsis treated with $20 \mu \mathrm{M}$ cycloheximide (Fig. 1b). Taken together, appearance of low molecular weight bands representing newly synthesized proteins were dependent upon puromycin treatment, but were largely absent during treatment with a protein synthesis inhibitor.

Although this study focused on root tissue, additional experiments sought to determine if SUnSET could also detect protein synthesis in leaves. Newly synthesized proteins could not be robustly detected in leaves when using the same conditions as for roots, i.e. $20 \mu \mathrm{M}$ puromycin treatment for $30 \mathrm{~min}$. However, newly synthesized proteins were visualized on a Western Blot when plants were treated with $50 \mu \mathrm{M}$ puromycin for 30 and $60 \mathrm{~min}$ (Fig. 1c). To determine if SUnSET can detect changes in protein synthesis in leaf tissue, plants were grown with or without cyclohexide for $4 \mathrm{~h}$, and subsequently treated with $50 \mu \mathrm{M}$ puromycin for $30 \mathrm{~min}$. Using SUnSET as described above, intensity of bands representing newly synthesized proteins were inversely correlated with cycloheximide concentration, indicating that SUnSET can also be used to monitor changes in protein synthesis in leaf tissue (Fig. 1d).

This study utilized the efficacy of SUnSET to monitor the effects of MG132-a potent proteasome inhibitoron protein synthesis in root tissue. Initial experiments were aimed at establishing evidence that $50 \mu \mathrm{M}$ MG132 inhibited the proteasome in the roots of Arabidopsis plants. As anticipated, proteasome inhibition caused by MG132 treatment increased levels of high molecular weight polyubiquitinated proteins after 4 and $16 \mathrm{~h}$ compared to control plants (Fig. 2a). MG132 treatment did not affect levels of Cox2, a subunit of the cytochrome-c oxidase complex. The proteasome degrades ubiquitinated proteins, and in yeast and animal cells its inhibition depletes the amino acid pool. Thus, it was important to determine if MG132 similarly affects amino acid levels in plants. Proteasome inhibition decreased levels of free amino acids by 55 and $43 \%$ after 4 and $16 \mathrm{~h}$, respectively (Fig. 2b). Prolonged proteasome inhibition in yeast 


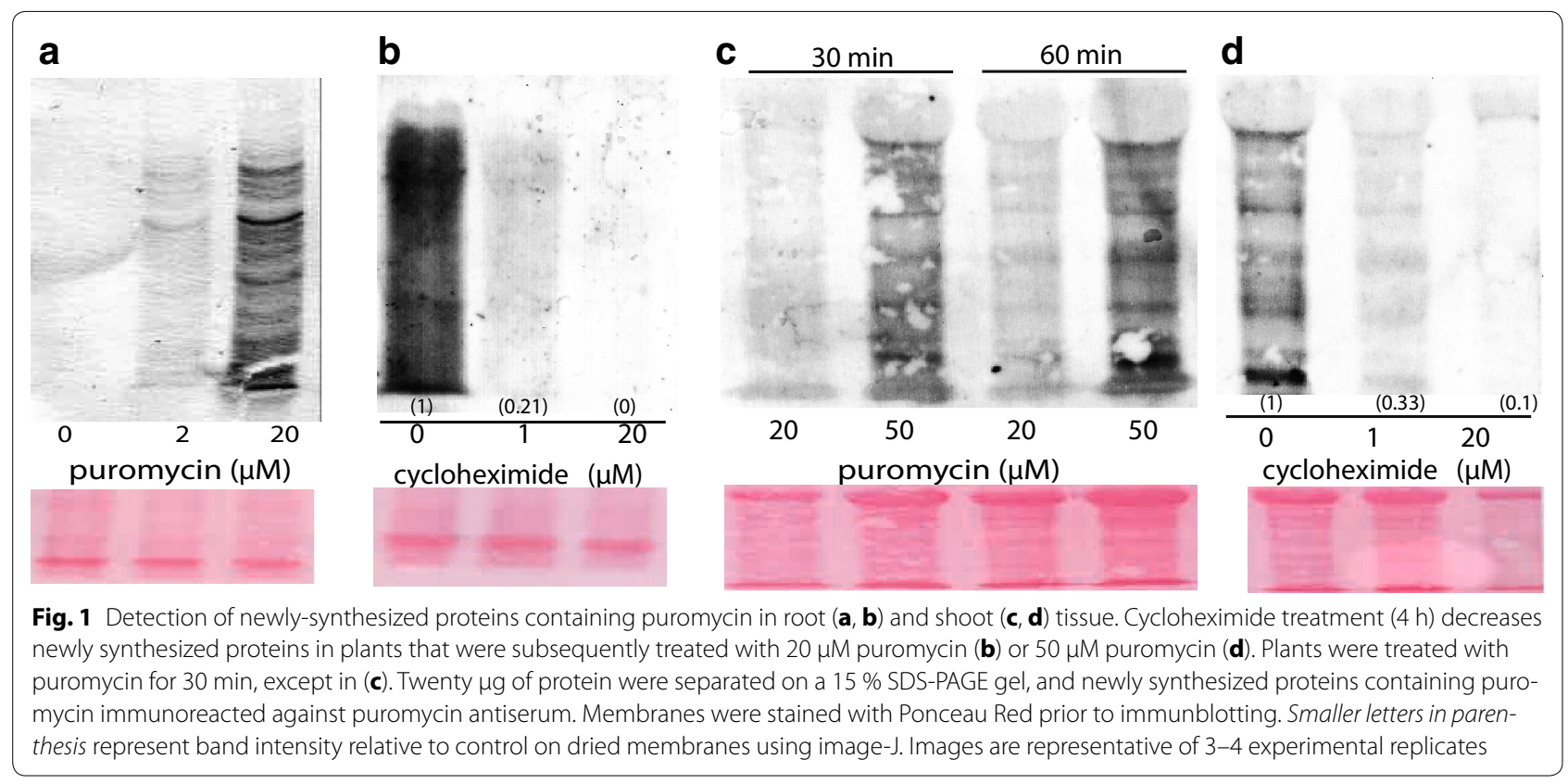

and animal cells results in lethality, and it was desirable to determine if MG132 treatment used in this study resulted in cell death. However, MG132 did not decrease cell viability as determined by fluorescence of fluorescein diacetate, a cell permeable probe that is indicative of membrane integrity (Fig. 2c). Thus, 50 MM MG132 inhibited the proteasome, as judged by the increase in polyubiquitinated proteins and the decrease in amino acids, but did not cause lethality.

The effect of MG132 on protein synthesis in Arabidopsis roots was estimated by treating Arabidopsis plants with $20 \mu \mathrm{M}$ puromycin for $30 \mathrm{~min}$. Newly synthesized proteins were nearly absent in MG132-treated plants after 4 and $16 \mathrm{~h}$; proteasome inhibition resulted in a faint banding pattern, but the intensity of these bands was greater compared to the negative control plants that were not treated with puromycin (Fig. 3), indicating that protein synthesis was not fully impaired by MG132. Ponceau red staining on the same membrane prior to blotting confirmed the presence of proteins. Therefore, the severe decrease in protein synthesis in MG132-treated plants could not be explained by a decrease in protein content.

The effects of proteasome inhibition are reversible in yeast and animal cells. SUnSET was used to determine if recovery from proteasome inhibition would restore levels of newly synthesized proteins. Plants treated with MG132 for $4 \mathrm{~h}$ were washed and placed in media without MG132 for 0.5 and $3 \mathrm{~h}$. MG132-treated plants allowed to recover for $3 \mathrm{~h}$ in control media had more newly synthesized proteins compared to MG132-treated plants (Fig. 4). Moreover, recovery from proteasome inhibition after $3 \mathrm{~h}$ resulted in higher rates of de novo protein synthesis compared to untreated plants. Increased protein synthesis after recovery was concomitant with a notable decrease in polyubiquitinated proteins, similar to control plants.

In addition to proteasome inhibition, experiments were conducted to determine if SUnSET could detect changes in protein synthesis that often occur during stress. Arabidopsis plants were grown in $0.1 \mathrm{M} \mathrm{NaCl}$ or the absence of sulfur for 4 days; these stress treatments have previously been reported to decrease protein synthesis. Compared to control plants grown on complete media for an additional 4 days, salt stress and sulfur starvation decreased protein synthesis, at or near similar levels of MG132treated plants after $4 \mathrm{~h}$ (Fig. 5).

\section{Discussion}

Protein synthesis is indispensible to cellular survival, but can be altered by stress [28] and developmental patterns. Methods to measure protein synthesis in plants have traditionally required a tracer. Stable isotopes such as $15-\mathrm{N}$ or 34-S can be used to gauge protein synthesis; although handling radioactivity is averted, analysis using stable isotopes is dependent upon a mass spectrometer which can be cost prohibitive. More commonly, radioactive isotopes such 35-S or 14-C are favored for both its precision and robustness, but come with risks. Recently, SUnSET techniques have been show to accurately asses protein synthesis in yeast and animal cells, and averts the health hazards associated with radioactivity.

This study represents the first time that SUnSET has been used to successfully monitor protein synthesis in 


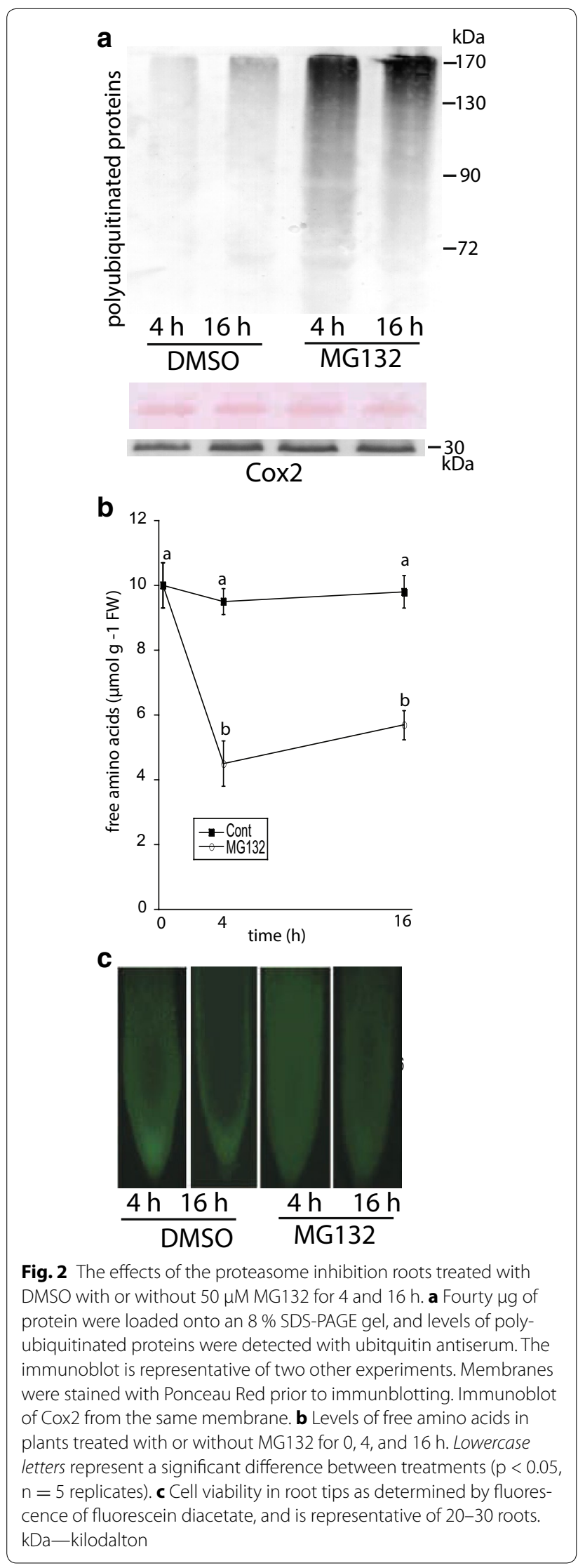

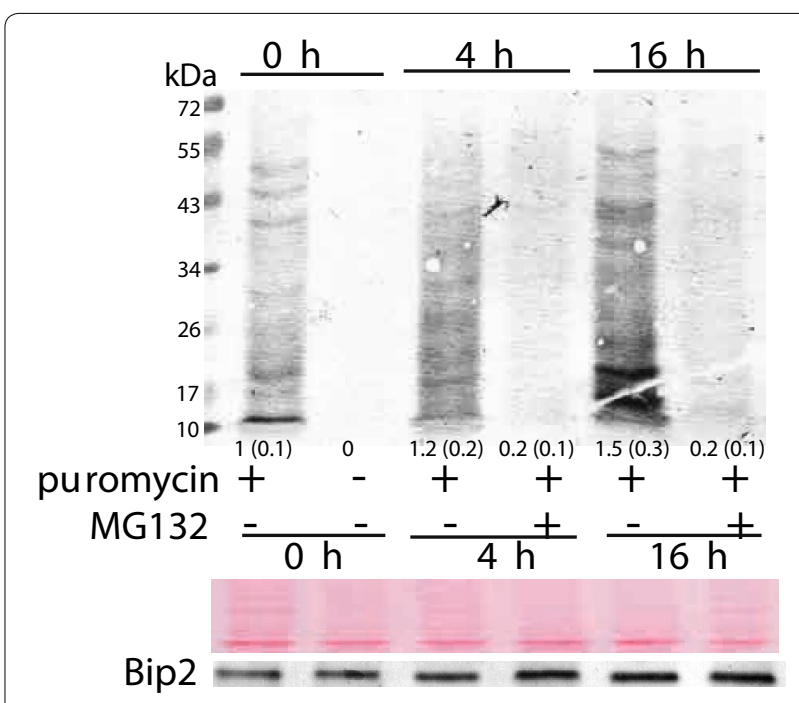

Fig. 3 Effects of MG132 on levels of newly synthesized proteins in roots using SUnSET. Plants were treated with or without $50 \mu \mathrm{M}$ MG132 and subsequently treated with or without $20 \mu \mathrm{M}$ puromycin. Twenty $\mu \mathrm{g}$ of root protein were separated on a $15 \%$ SDS-PAGE gel, and newly synthesized proteins were detected using puromycin antiserum. Membranes were stained with Ponceau Red prior to immunblotting. Smaller letters in parenthesis represent band intensity relative to control on dried membranes using image-J. Images are representative of four experimental replicates. Levels of Bip2 were analyzed from the same membrane. $\mathrm{kDa}$ - kilodalton

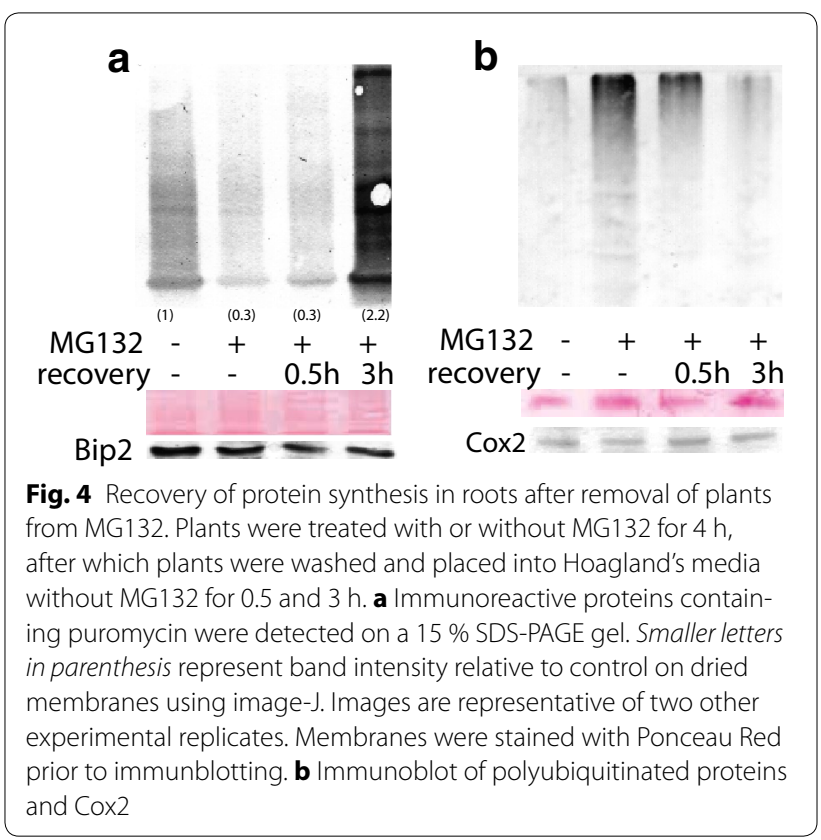

plants. Therefore, this non-radioactive method is suitable to detect changes in protein synthesis in plants. This conclusion is warranted on the grounds that treatment with the protein synthesis inhibitor cycloheximide 


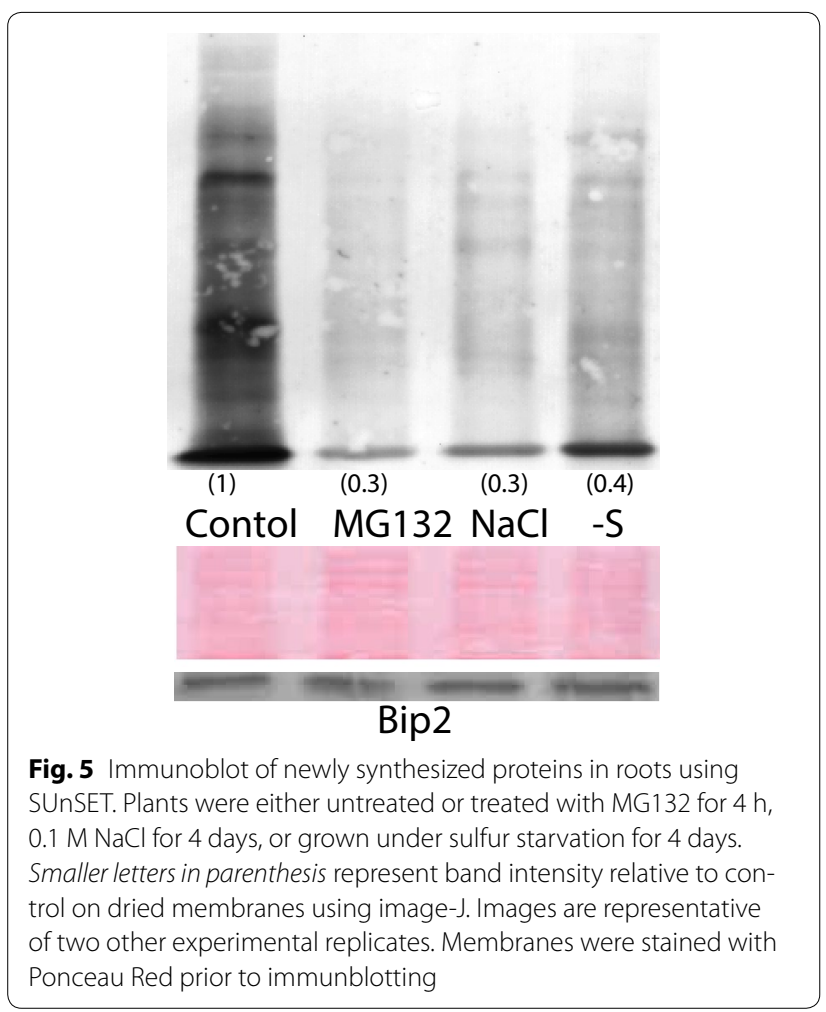

severely decreased levels of newly synthesized proteins, suggesting that puromycin only detected de novo protein synthesis in roots and leaves. Studies comparing SUnSET and radioactive methods to detect changes in protein synthesis have observed nearly identical results [21], with an r-correlation of 0.9 being reported in one study [22]. Although this study did not compare decreased protein synthesis using SUnSET and traditional radioactive techniques, the data indicate that SUnSET can be a useful method to gauge changes in protein synthesis in plants. Lastly, this study used electrophoresis to detected newly synthesized proteins; however, protein synthesis using SUnSET has also been monitored using immunohistochemistry and confocal microscopy [21, 24]. Although it is probable that protein synthesis using SUnSET can be monitored in single plant cells or intact roots via microscopy, this remains to be experimentally confirmed.

The efficacy of SUnSET was utilized to demonstrate that proteasome inhibition in Arabidopsis roots decreased levels of newly synthesized proteins, which coincided with depletion of the amino acid pool. Although these results are in agreement with other studies in yeast and mammalian cells [7], it nonetheless represents the first time that the effects of proteasome inhibition on amino acids and protein synthesis have been reported in plants. MG132 reduced protein synthesis by $70-80 \%$. It is possible that the deleterious effects of MG132 on protein synthesis reported in this study is underestimated, because the proteasome can remove truncated proteins containing puromycin [29].

Recently it was discovered that Arabidopsis plants initiate autophagy in response to proteasome inhibition, likely in order to replenish the amino acid pool [19]. Data presented in this study suggest that autophagy is not able to overcome the detrimental effects of MG132 treatment and restore amino acid to homeostatic levels. Although autophagy is unlikely to maintain protein synthesis during proteasome inhibition, removal of MG132 after 4-h treatment caused an accumulation of newly synthesized proteins compared to untreated plants. These results suggest that plant roots can recover from proteasome inhibition and support elevated rates of de novo protein synthesis. These results mirror the increased de novo protein synthesis in plants after a recovery from nutrient deprivation [30, 31]. A similar recovery from proteasome inhibition also occurs in neurons [32], as the removal of MG132 increased protein synthesis more than twofold compared in untreated samples. Therefore, the effects of proteasome inhibition caused by MG132 appear to be reversible in roots, as first reported in yeast [33].

To determine whether or not SUnSET provides a fast and reliable means to monitor protein synthesis during adverse environmental conditions, Arabidopsis plants were subjected to salt stress and sulfur starvation. Both of these stressors reduced levels of newly synthesized proteins, which is in agreement with other studies that examined the effects of salt stress [34] and sulfur starvation [35]. These data indicate that SUnSET is applicable to detect dramatic changes in protein synthesis, which may eventually aid both physiological and developmental studies.

\section{Conclusions}

For the first time in plants, SUnSET was used to monitor changes in protein synthesis. As anticipated, decreased protein synthesis was observed in the roots and shoots of Arabidopsis plants treated with cycloheximide. MG132 also decreased protein synthesis in roots, but recovery from proteasome inhibition resulted in increased levels of newly synthesized proteins. Additionally, plants subjected to stress-including salt stress and sulfur starvation-also displayed a decrease in newly synthesized proteins. Collectively, these data are in good agreement with previous studies that have measured protein synthesis using radioactive materials. In this study, newly synthesized proteins were detected on a Western blot, which makes this method extremely cost effective and safer compared to methods requiring radioactive isotopes. Additionally, the protocol for SUnSET can easily be performed in $<12 \mathrm{~h}$. Therefore, in addition to a wide 
array of disciplines in plant biology, the method is suitable in educational settings where the use of radioactivity is restricted.

\section{Author details}

${ }^{1}$ Biology Department, Coastal Carolina University, Conway, SC 29526, USA.

2 Present Address: Ankara University, Biotechnology Institute, Tandoğan Campus, 06110 Ankara, Turkey.

\section{Acknowledgements}

The author thanks John Yewdell for correspondence during the initial stages of this project. This research was supported by the NSF-RUI program (MCB-1244009).

\section{Competing interests}

The author declares that there is no competing interests.

Received: 1 February 2016 Accepted: 9 March 2016

Published online: 16 March 2016

\section{References}

1. Coux O, Tanaka K, Goldberg AL. Structure and functions of the $20 \mathrm{~S}$ and 265 proteasomes. Ann Rev Biochem. 1996;65:801-47.

2. Smalle J, Vierstra RD. The ubiquitin $26 \mathrm{~S}$ proteasome proteolytic pathway. Ann Rev Plant Biol. 2004;55:555-90.

3. Yates $G$, Sadanandom A. Ubiquitination in plant nutrient utilization. Front Plant Sci. 2013:4:452.

4. Santner A, Estelle M. The ubiquitin-proteasome system regulates plant hormone signaling. Plant J. 2010;61:1029-40.

5. Schubert U, Anton LC, Gibbs J, Norbury CC, Yewdell JW, Bennink JR. Rapid degradation of a large fraction of newly synthesized proteins by proteasomes. Nature. 2000;404:770-4.

6. Vabulas RM, Hartl FU. Protein synthesis upon acute nutrient restriction relies on proteasome function. Science. 2005;310:1960-3.

7. Suraweera A, Münch C, Hanssum A, Bertolotti A. Failure of amino acid homeostasis causes cell death following proteasome inhibition. Mol Cell. 2012:48:242-53

8. Jiang HY, Wek RC. Phosphorylation of the a-subunit of the eukaryotic initiation factor-2 (elF2a) reduces protein synthesis and enhances apoptosis in response to proteasome inhibition. J Biol Chem. 2005;280:14189-202.

9. Ding Q, Dimayuga E, Markesbery WR, Keller JN. Proteasome inhibition induces reversible impairments in protein synthesis. FASEB J. 2006;20:1055-63.

10. Cowan JL, Morley SJ. The proteasome inhibitor, MG132, promotes the reprogramming of translation in C2C12 myoblasts and facilitates the association of hsp25 with the elF4F complex. Euro J Biochem. 2004;271:3596-611.

11. Chung KK, Dawson VL, Dawson TM. The role of the ubiquitin-proteasomal pathway in Parkinson's disease and other neurodegenerative disorders. Trends Neurosci. 2001:24:7-14.

12. Groll M, Schellenberg B, Bachmann AS, Archer CR, Huber R, Powell TK, Dudler R. A plant pathogen virulence factor inhibits the eukaryotic proteasome by a novel mechanism. Nature. 2008;452:755-8.

13. Karmous I, Chaoui A, Jaouani K, Sheehan D, El Ferjani E, Scoccianti V, Crinelli R. Role of the ubiquitin-proteasome pathway and some peptidases during seed germination and copper stress in bean cotyledons. Plant Physiol Biochem. 2014;76:77-85.

14. Pena LB, Zawoznik MS, Tomaro ML, Gallego SM. Heavy metals effects on proteolytic system in sunflower leaves. Chemosphere. 2008;72:741-6.

15. Lee BD, Hwang S. Overexpression of NtUBQ2 encoding Ub-extension protein enhances cadmium tolerance by activating $20 \mathrm{~S}$ and 265 proteasome in tobacco (Nicotiana tabacum). Acta Physiol Plant. 2015;37:1-8.

16. Wang S, Kurepa J, Hashimoto T, Smalle JA. Salt stress-induced disassembly of Arabidopsis cortical microtubule arrays involves
26S proteasome-dependent degradation of SPIRAL1. Plant Cell. 2011;23:3412-27.

17. Vallentine P, Hung CY, Xie J, Van Hoewyk D. The ubiquitin-proteasome pathway protects Chlamydomonas reinhardtii against selenite toxicity, but is impaired as reactive oxygen species accumulate. AoB Plants. 2014;6:plu062.

18. Dimkovikj A, Van Hoewyk D. Selenite activates the alternative oxidase pathway and alters primary metabolism in Brassica napus roots: evidence of a mitochondrial stress response. BMC Plant Biol. 2014;14:259. doi:10.1186/s12870-014-0259-6.

19. Marshall RS, Li F, Gemperline DC, Book AJ, Vierstra RD Autophagic degradation of the 265 proteasome is mediated by the dual ATG8/ubiquitin receptor RPN10in Arabidopsis. Mol Cell. 2015:58:1053-66.

20. Goodman CA, Hornberger TA. Measuring protein synthesis with SUnSET: a valid alternative to traditional techniques? Exerc Sport Sci Rev. 2013;41:107.

21. Schmidt EK, Clavarino G, Ceppi M, Pierre P. SUnSET, a nonradioactive method to monitor protein synthesis. Nat Methods. 2009;6:275-7.

22. Goodman CA, Mabrey DM, Frey JW, Miu MH, Schmidt EK, Pierre P, Hornberger TA. Novel insights into the regulation of skeletal muscle protein synthesis as revealed by a new nonradioactive in vivo technique. FASEB J. 2011:25:1028-3109.

23. Hoeffer CA, Santini E, Ma T, Arnold EC, Whelan AM, Wong H, Pierre P, Pelletier J, Klann E. Multiple components of elF4F are required for protein synthesis-dependent hippocampal long-term potentiation. J Neurophysiol. 2013;109:68-76.

24. Liu J, Xu Y, Stoleru D, Salic A. Imaging protein synthesis in cells and tissues with an alkyne analog of puromycin. Proc Natl Acad Sci USA 2012;109:413-8

25. David A, Dolan BP, Hickman HD, Knowlton JJ, Clavarino G, Pierre P, Bennink JR, Yewdell K. Nuclear translation visualized by ribosome-bound nascent chain puromycylation. J Cell Biol. 2012;197:45-57.

26. Sabbagh M, Van Hoewyk D. Malformed selenoproteins are removed by the ubiquitin-proteasome pathway in Stanleya pinnata. Plant Cell Physiol. 2012;53:555-64.

27. Magne C, Larher F. High sugar content of extracts interferes with colorimetric determination of amino acids and free proline. Anal Biochem. 1992;200:115-8

28. Dubey RS. Protein synthesis by plants under stressful conditions. Handb Plant Crop Stress. 1999:19:365-97.

29. Lacsina JR, Marks OA, Liu X, Reid DW, Jagannathan S, Nicchitta CV Premature translational termination products are rapidly degraded substrates for MHC class I presentation. PLoS One. 2012;7:e51968.

30. Zhang Q, Lee BR, Park SH, Zaman R, Avice JC, Ourry A, Kim TH. Sulfate resupply accentuates protein synthesis in coordination with nitrogen metabolism in sulfur deprived Brassica napus. Plant Physiol Biochem. 2015;28(87):1-8

31. Scheible WR, Morcuende R, Czechowski T, Fritz C, Osuna D, Palacios-Rojas N, Schindelasch D, Thimm O, Udvardi MK, Stitt M. Genome-wide reprogramming of primary and secondary metabolism, protein synthesis, cellular growth processes, and the regulatory infrastructure of Arabidopsis in response to nitrogen. Plant Physiol. 2004;136:2483-99.

32. Ding $\mathrm{Q}$. Dimayuga $E$, Markesbery WR, Keller JN. Proteasome inhibition induces reversible impairments in protein synthesis. FASEB J. 2006:20:1055-63.

33. Lee $\mathrm{DH}$, Goldberg AL. Selective inhibitors of the proteasome-dependent and vacuolar pathways of protein degradation in Saccharomyces cerevisiae. J Biol Chem. 1996;271:27280-4.

34. Greenway H, Munns R. Mechanisms of salt tolerance in non-halophytes. Ann Rev Plant Physiol. 1980;31:149-90.

35. Zhang Q, Lee BR, Park SH, Zaman R, Avice JC, Ourry A, Kim TH. Sulfate resupply accentuates protein synthesis in coordination with nitrogen metabolism in sulfur deprived Brassica napus. Plant Physiol Biochem. 2015;87:1-8. 